\title{
CRITICAL BEHAVIOR OF INTERFACES: ROUGHENING AND WETTING PHENOMENA
}

\author{
REINHARD LIPOWSKY, STEFAN GROTEHANS, and GÖTZ J.O. SCHMIDT \\ Institut für Festkörperforschung, Forschungszentrum Jülich \\ Postfach 1913, D-5170 Jülich, Germany
}

\section{ABSTRACT.}

The critical behavior of interfaces is discussed from a theoretical point of view. Two classes of critical phenomena will be considered: (i) Roughening phenomena related to changes in the interfacial morphology; and (ii) Wetting phenomena related to changes in the interfacial structure. In two dimensions, the critical behavior can be determined exactly for a variety of models. As a result, one obtains different universality classes depending on the nature of the intermolecular forces.

\section{Introduction}

Critical phenomena at interfaces have been studied, both theoretically and experimentally, for many years. Here, we will discuss two classes of critical phenomena related to the reduced dimensionality of the interface. ${ }^{1}$

In some cases, the interface can simply be viewed as a planar 2-dimensional system. However, it can also 'bulge' into the third dimension and then attain nonplanar morphologies. This roughening of the interface can be thermally excited or induced by frozen randomness. In addition, the interface has a certain depth profile and thus has itself a third dimension. This intrinsic thickness of the interface can become mesoscopic as in wetting phenomena; one then has a system which interpolates between two and three dimensions.

This paper is organized as follows. First, the physics of roughening and wetting is briefly reviewed (Sect. 2). The corresponding critical behavior at roughening and wetting can be studied in the framework of effective interface models (Sect. 3). The last section focusses on the behavior in two dimensions: a variety of critical effects will be discussed arising from the behavior of 1-dimensional interfaces or domain boundaries. These effects should be accessible to experiments, for example in the context of Langmuir-Blogdett films.

\section{Roughening and Wetting Phenomena}

\subsection{Interfacial morphology and roughening}

An interface or domain wall represents the contact region between two bulk phases of matter. Its macroscopic shape is governed by the interfacial free energy or tension, $\Sigma$, which is the work (per unit area) required to create new interfacial area. If the two bulk phases, say $\alpha$ and $\beta$, are both isotropic fluids, $\Sigma$ is also isotropic, and a macroscopic chunk of $\beta$ phase surrounded by $\alpha$ phase has a spherical shape (if one can ignore the effects of gravity). If one of the two phases is anisotropic, the tension $\Sigma$ is anisotropic as well and depends on the interfacial orientation. For crystalline phases, this anisotropy leads to the possibility of flat interfaces or facets.

From the theoretical point of view, the equilibrium shape of crystals is completely determined, up to a scale factor, by the functional dependence of the interfacial (or surface) free energy, $\Sigma$, on the interfacial orientation or normal vector, $\hat{n}$. Now, assume that $\Sigma=\Sigma(\hat{n})$ has a local minimum for a certain symmetry direction, $\hat{n}=\hat{n}_{0}$, and consider interfaces with a small tilt angle, $\theta=\cos ^{-1}\left(\hat{n} \cdot \hat{n}_{0}\right)$. If the orientation with $\theta=0$ corresponds to a smooth interface or facet, one has ${ }^{2}$

$$
\Sigma(\theta) \approx \Sigma_{0}+\Sigma_{S}|\theta| \quad \text { for small } \theta
$$

Mat. Res. Soc. Symp. Proc. Vol. 237. (C)1992 Materials Research Society 
where $\Sigma_{S}$ represents the step free energy (per unit length and unit height).

As the temperature $T$ is increased, $\Sigma_{S}$ may vanish at a characteristic roughening temperature, $T=T_{*}$. On a macroscopic scale, this implies that the facet shrinks and finally disappears from the equilibrium crystal shape at $T=T_{*}$. On a microscopic scale, the roughening of a facet implies a proliferation of more and more steps or ledges on the facet. ${ }^{3}$ In general, facets corresponding to different symmetry directions have different step free energies and, thus, different roughening temperatures. For example, three distinct roughening temperatures corresponding to three different facets have been experimentally observed for $\mathrm{He}^{4}$ crystals.

Above the roughening temperature, i.e., for $T>T_{*}$, the step free energy vanishes, and the interface is rough. For a periodic crystal, the interfacial free energy then behaves as ${ }^{2}$

$$
\Sigma(\theta) \approx \Sigma_{0}+\frac{1}{2} \Sigma_{2} \theta^{2} \quad \text { for small } \theta
$$

Somewhat surprisingly, this behavior applies to periodic crystals even in the presence of quenched impurities (or frozen randomness). These systems are charcterized by a finite interfacial stiffness, $\bar{\Sigma}$, which is defined by $\tilde{\Sigma} \equiv \Sigma_{0}+\Sigma_{2}$. On the other hand, for a quasiperiodic crystal, the interfacial free energy can exhibit a more complicated behavior given by $\Sigma(\theta) \approx \Sigma_{0}+\frac{1}{k} \Sigma_{k}|\theta|^{k}$ for small $\theta$ with $1<k \leq 2$. $^{4}$

\subsection{Interfacial structure and wetting}

So far, we have tacitly assumed that the interface between the two bulk phases, say $\alpha$ and $\gamma$, has a microscopic width. Thus, the densities of the system when viewed with a local probe vary on a microscopic scale which is set by the size of the molecules.

Now, let us change a thermodynamic field, e.g. temperature or pressure, in such a way that the system approaches a triple point where a third phase, $\beta$, can coexist with the two phases $\alpha$ and $\gamma$. Then, in thermal equilibrium, a layer of the $\beta$ phase may appear in the $\alpha \beta$ interface. As one comes closer and closer to the triple point, the thickness of this intermediate layer may continuously grow: this is the case of complete wetting. On the other hand, no layer may appear in the $\alpha \beta$ interface or the thickness of this layer may saturate and remain finite as the triple point is attained: this is the case of incomplete wetting.

The presence of a wetting layer leads to a new length scale, $\ell$, which is given by the wetting layer thickness, i.e., by the separation of the two interfaces bounding this layer. This scale has been measured in many experiments. In the case of complete wetting, it typically varies from the molecular scale far from the triple point up to hundreds of $\mathrm{nm}^{\prime}$ 's sufficiently close to the triple point. In principle, the thickness $\ell$ diverges as the triple point is attained.

The situation just described corresponds to the case of full chemical equilibrium between the $\beta$ layer and the two bulk phases: all three phases exchange their molecules and are thus characterized by the same values of the chemical potentials. This type of chemical equilibrium usually applies to wetting in fluid mixtures. ${ }^{5}$ Some wetting systems exhibit only partial chemical equilibrium in which the $\beta$ layer chemically equilibrates only with one of the two bulk phases on experimentally relevant time scales. The other bulk phase, say $\gamma$, then plays the role of an inert 'spectator' phase. One example is multilayer adsorption of small molecules onto a solid. ${ }^{6}$ In the latter case, the solid substrate does not evaporate on the time scales of typical experiments. Finally, one may also study the situation where the $\beta$ layer does not exchange particles with any of the two bulk phases. This case of hindered or blocked chemical equilibrium applies to sufficiently fast dynamic processes such as, e.g., dry spreading. ${ }^{7}$

Wetting has to be distinguished from heterogeneous nucleation even though the nucleation barrier is intimately related to the wetting properties. Indeed, heterogeneous nucleation 
at the interface occurs when the system has passed the triple point and the bulk phases, $\alpha$ and $\gamma$, (or at least one of them) have become thermodynamically unstable. The energy barrier for nucleation of $\beta$ droplets is reduced at the interface. For complete wetting, this barrier is, in fact, reduced to zero, and the transformation into the stable $\beta$ phase will start at the interface or surface.

\section{Theoretical description}

\subsection{Critical behavior}

\subsubsection{Roughness exponent $\zeta$}

In many cases, interfaces are rough, i.e., they make large transverse excursions from their mean or average position. More precisely, an interface is rough if the typical size, $\xi_{\perp}$, of its transverse excursions grows with its size, $\xi_{\|}$. This behavior can usually be described by the scaling law

$$
\xi_{\perp} \sim \xi_{\|}^{\zeta}
$$

which defines the roughness exponent $\zeta$. The universality classes for this exponent are primarily determined by the symmetry of the two bulk phases adjacent to the interface. This will be explicitly discussed below for the case of periodic and quasi-periodic systems.

\subsubsection{Roughening exponent $\nu_{\perp}$}

The roughness of the interface can change as a function of temperature or any other control parameter. For example, the 2-dimensional interface between a periodic crystal and its vapor is smooth at low temperatures, $T$, with $\xi_{\perp}$ of the order of the lattice parameter, $a$, and thus $\zeta=0$, but may become rough at sufficiently large $T$. This roughening can be understood in terms of the effective potential for the interface arising from the crystal lattice.

At low temperatures, a sufficiently strong potential will be able to localize the interface and thus to suppress its excursions on large scales. As the temperature is increased, the interfacial fluctuations become more pronounced and may eventually overcome the confining potential. At this point, the interface undergoes a roughening transition.

As the roughening temperature, $T_{*}$, is approached from below, the interfacial roughness, $\xi_{\perp}$, grows as

$$
\xi_{\perp} \sim 1 /\left(T_{*}-T\right)^{\nu_{\perp}}
$$

which defines the roughening exponent $\nu_{\perp}$.

In general, the roughening exponent $\nu_{\perp}$ depends on the nature of the effective potential confining the interface. This will be shown below for the roughening (or delocalization) transitions in the presence of a linear defect (such as a grain boundary).

\subsubsection{Wetting exponent $\psi$}

Now consider a thin wetting layer bounded by two interfaces. In this case, the interfaces experience a mutual interaction potential. Two different cases must be distinguished: (i) At $(\alpha \beta \gamma)$-coexistence (compare Sect. 2.2), the bulk free energies of all three phases $\alpha, \beta$, and $\gamma$ are equal and the interaction potential decays to zero for large separations, $\ell$, of the two interfaces; and (ii) As the triple point is approached along $(\alpha \gamma)$-coexistence, the $\beta$ phase represents a metastable thermodynamic phase which has a bulk free energy $\hat{f}_{\beta}$ which is larger than the bulk free energy $f_{\alpha \gamma}$ of the two other phases. Thus, the excess free energy per unit area of the layer contains a term $\left(\hat{f}_{\beta}-f_{\alpha \gamma}\right) \ell$ and the effective interaction between the interfaces grows linearly $\sim \ell$ for large $\ell$.

In case (i), i.e., along a line triple points, the system can undergo a wetting transition at a characteristic wetting temperature, $T_{*}$. If this transition is continuous, the mean separation 
$\langle\ell\rangle$ which gives the layer thickness usually diverges as

$$
\langle\ell\rangle \sim 1 /\left(T_{*}-T\right)^{\psi}
$$

which defines the wetting exponent $\psi$. In general, this exponent depends on the nature of the interaction potential experienced by the two interfaces, see below.

\subsection{Effective interface models}

The simplest model with two coexisting phases is the lattice gas or Ising model with nearest-neighbor interactions and appropriate boundary conditions. For roughening phenomena, one can consider an Ising model where half of the boundary spins points up and the other half points down. In this way, an interface is enforced running across the system.

For wetting phenomena, one would like to consider the coexistence of three phases. This can be done in the context of semi-infinite Ising models in which the 'empty space' adjacent to the surface plays the role of the third phase. Physically, this corresponds to the case of partial chemical equilibrium in which the wetting layer exchanges molecules only with one of the two adjacent bulk phases.

In the continuum limit, the interfacial position can be described by a displacement field $\ell=\ell(x)$ where $\mathrm{x}$ is a $(\mathrm{d}-1)$-dimensional coordinate parallel to a reference plane. For roughening, the field $\ell$ gives the distance of the interface from this reference plane; for wetting, it measures the separation of the two interfaces bounding the wetting layer.

The effective Hamiltonian for the interfacial displacement field $\ell$ has the generic form ${ }^{8,9}$

$$
\mathcal{H}\{\ell\}=\int d^{d-1} x\left\{\frac{1}{2} \tilde{\Sigma}(\nabla \ell)^{2}+V(\ell)\right\}
$$

where $\tilde{\Sigma}$ is an appropriate interfacial stiffness and $V(\ell)$ is an external or interaction potential for the interface. In the following, we will focus on the case $d=2$.

For roughening phenomena, the potential $V(\ell)$ describes the effect of the underlying lattice. For a periodic and for a quasi-periodic lattice, this potential will be periodic and quasi-periodic, respectively. We will also discuss the influence of a line (or plane) of defects which acts to localize the interface. In this latter case, the potential $V(\ell)$ has local minima at the position of the defect and for $\ell=\infty$. In most cases, the potential for roughening is taken to be symmetric, i.e., $V(\ell)=V(-\ell)$; one exception is provided by the quasi- periodic Fibonacci-potential.

For wetting phenomena, the potential $V(\ell)$ describes the interaction of the two interfaces at separation $\ell$. It then contains a hard wall $\ell=0$ which ensures that $\ell \geq 0$ since the two interfaces cannot interpenetrate one another. As mentioned above, the potential $V(\ell)$ has the generic form

$$
V(\ell)=H \ell+V_{D I}(\ell) \quad \text { for } \quad \ell>0
$$

where $H=\hat{f}_{\beta}-f_{\alpha \gamma}$ measures the distance from $(\alpha \beta \gamma)$ coexistence and $V_{D r}(\ell)$ describes the direct interaction arising from molecular interactions. This direct interaction decays to zero for large $\ell$.

\section{Critical Behavior in Two Dimensions}

Now, consider a 2-dimensional system in which two thermodynamic phases coexist. One simple example is the 2-dimensional Ising model in which domains of up-spins can coexist with domains of down-spins. As mentioned, the morphology of an interface separating two such domains depends on the underlying symmetry of the system. Two cases will be briefly discussed: (i) Periodic systems; and (ii) Quasi-periodic systems. 
The motivation for the discussion of these 2-dimensional systems is three-fold: (i) For many theoretical models, the critical behavior in two dimensions can be determined exactly. In this way, one can check theoretical ideas and concepts which should be valid in general; (ii) 2-dimensional systems are easily accessible to computer simulations which provides an additional theoretical check; and (iii) These effects can be observed in careful experiments which probe the lateral structure within a 2 -dimensional interface or surface. ${ }^{10}$

\subsection{Periodic systems}

\subsubsection{Roughening transition from a line of defects}

First, consider a periodic lattice in two dimensions. It is well-known that the interface running through such a system is rough at any finite temperatures, $T>0$. In fact, the roughness exponent has the universal value $\zeta=1 / 2$.

Now consider a periodic system which contains a line of defects. In general, this line will lead to an effective interface potential which is localized around the defect. We will consider the case where this potential has a short-ranged and a long-ranged part. More precisely, the short-ranged part is taken to be a simple square well as given by ${ }^{11}$

$$
V(\ell)=U \quad \text { for } \quad-\ell_{o} \leq \ell \leq \ell_{o}
$$

while the long-ranged part behaves as

$$
V(\ell)=-W / \ell^{2} \quad \text { for } \quad \ell>\ell_{o}
$$

Such a model can be studied by transfer matrix methods. This leads to a 1-dimensional Schrödinger-type equation for which the interaction $V(\ell)$ plays the role of a quantummechanical potential. It is convenient to consider the dimensionless interaction $v(z) \equiv 2 \tilde{\Sigma} \ell_{0}^{2}$ $V\left(\ell_{0} z\right) / T^{2}$ where $\bar{\Sigma}$ is the stiffness of the interface. This rescaled potential then depends on the two dimensionless parameters

$$
u=2 \tilde{\Sigma} \ell_{o}^{2} U / T^{2} \quad \text { and } \quad w=2 \dot{\Sigma} W / T^{2}
$$

In terms of these variables, one finds a whole line of roughening transitions as displayed in Fig. $1 .{ }^{11}$ The locus of transitions consists of three different subregimes (A), (B), and (C), see Fig. 1.

It is important to note that a change in temperature $T$ corresponds to a straight line in Fig. 1 which approaches the origin with $(u, w)=(0,0)$ for large $T$. Thus, for $U \leq 0$ and/or $W \leq 0$, i.e., if both parts of the defect potential are attractive, the interface is smooth and localized for any finite $T$ and the roughening transition occurs at $T_{*}=\infty$ corresponding to $(u, w)=(0,0)$. In contrast, if the two amplitudes $U$ and $W$ of the short-ranged and longranged potential parts have different sign, the $T$-trajectory intersects the locus of transitions at a finite transition temperature, $T=T_{*}<\infty$.

Subregime (A) is located at negative values of $w$. For $w<-1 / 4$, there is no roughening transition and the interface is always localized by the long-ranged attractive part of the potential. As $w=-1 / 4$ is approached from below, the interface undergoes a roughening transition of infinite order provided the amplitude $u$, of the short-ranged potential is larger 


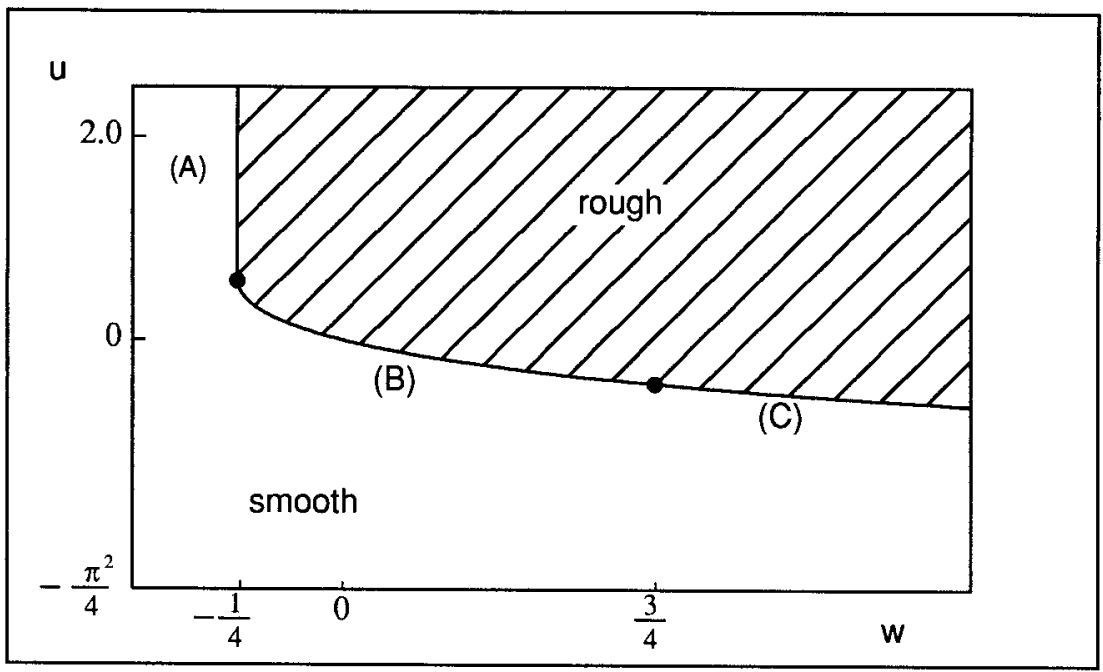

Figure 1: Line of roughening transitions which consists of three regimes (A), (B), and (C). The parameters $w$ and $u$ are defined in (4.3).

than a critical value, $u_{m c}=0.595$. At this transition, the interfacial roughness $\xi_{\perp}$, behaves as

$$
\xi_{\perp} \sim \exp [\pi / \sqrt{-w-1 / 4}]
$$

The roughening transitions in subregime (B) are located at $-1 / 4<w<3 / 4$. At these transitions, the interfacial roughness diverges as

$$
\xi_{\perp} \sim 1 /\left(T_{*}-T\right)^{\nu_{\perp}}
$$

with the $w$-dependent roughening exponent

$$
\nu_{\perp}=1 / \sqrt{1+4 w} .
$$

The case $w=0$ belongs to subregime $(B)$ and corresponds to all potentials which decay faster than $1 / \ell^{2}$ for large $\ell$. This is a rather large class of defect potentials. For such potentials, the roughening transition occurs at $T_{*}=\infty$ or $u_{*}=0$. The corresponding critical behavior is given by

$$
\xi_{\perp} \sim 1 / u^{\nu_{\perp}} \sim T^{2 \nu_{\perp}} \quad \text { with } \quad \nu_{\perp}=1
$$

where the universal value of $\nu_{\perp}$ is obtained from (4.6) for $w=0$.

The roughening transitions within subregime (C) are located at $w>3 / 4$. In this case, one finds that the interfacial roughness still diverges as in (4.5), i.e., as a power law but with

$$
\begin{aligned}
\nu_{\perp} & =1-(1 / 4) \sqrt{1+4 w} & & \text { for } 3 / 4<w<15 / 4 \\
& =0 & & \text { for } 15 / 4<w .
\end{aligned}
$$


Thus, subregime (C) consists itself of several regimes. The value $\nu_{\perp}=0$ for $w>15 / 4$ corresponds to a discontinuous behavior of $\xi_{\perp}$ which jumps at $T_{*}$ from a finite value to infinity. However, higher moments of $\ell$ still diverge in a continuous fashion as explained in the next paragraph.

Indeed, the different character of the roughening transition for $w<3 / 4$ and $w>3 / 4$ can be seen more clearly if one considers the moments, $\left\langle\ell^{n}\right\rangle$, of arbitrarily high order $n$. In subregime $(\mathrm{B})$, one has

$$
\left\langle\ell^{n}\right\rangle \sim \xi_{\perp}^{n} \sim \xi_{\|}^{n / 2}
$$

with

$$
\xi_{\|} \sim 1 /\left(T_{*}-T\right)^{\nu_{\|}} \quad \text { and } \quad \nu_{\|}=2 / \sqrt{1+4 w}
$$

as follows from (4.5) and (4.6) and the scaling relation $\xi_{1} \sim \xi_{\|}^{\zeta}$ with $\zeta=1 / 2$. In subregime (C), on the other hand, the moments behave as

$$
\left\langle\ell^{n}\right\rangle \sim \bar{P}\left(\xi_{\|}^{\zeta}\right) \xi_{\|}^{n / 2}
$$

with

$$
\xi_{\|} \sim 1 /\left(T_{*}-T\right)^{\nu_{\|}} \quad \text { and } \quad \nu_{\|}=1
$$

where $\bar{P}\left(\xi_{\|}^{\zeta}\right)$ is the probability that the interfacial separation $\ell$ is of the order of $\xi_{\|}^{\zeta}$. In the limit of large $\xi_{\|}$, this probability scales as ${ }^{12}$

$$
\bar{P}\left(\xi_{\|}^{\zeta}\right) \sim \xi_{\|}^{1-\mu} \quad \text { with } \quad \mu=\sqrt{w+1 / 4}
$$

This behavior has a rather simple interpretation in terms of interfacial humps. As in (3.1), interfacial humps with wavelength $\xi_{\|}$have a transverse extension $\xi_{\perp} \sim \xi_{\|}^{\zeta}$ with $\zeta=1 / 2$ for all values of $w$. However, these humps represent typical fluctuations in subregime (B) but exceptional fluctuations in subregime (C). ${ }^{12}$ In the latter case, two $\xi_{\|}$-humps are separated by a weakly fluctuating segment of linear size $\sim \xi_{\|}^{\mu}$. Then the interfacial free energy per unit length has a singular contribution $f_{I} \sim T / \xi_{\|}^{\mu}$.

\subsubsection{Wetting}

Wetting in two dimensions can be studied in the framework of a semi-infinite Ising model with nearest-neighbor couplings. A wetting transition is obtained if the coupling constants between the first two rows of spins are weaker than the coupling constants in the bulk. ${ }^{13}$

For such a system, the effective interaction $V(\ell)$ between the interface and the boundary (or surface) is given by a square-well potential with a hard-wall condition, $V(\ell)=\infty$ for $\ell<0$. In general, long-ranged couplings between the spins or long-ranged surface fields will lead to a long-ranged part of $V(\ell)$.

In semi-infinite Ising models, one has only two coexisting phases and the third phase is represented by the 'empty' half-space. In addition, one of the two interfaces bounding the wetting layer is given by the boundary of the lattice which does not fluctuate and thus corresponds to an interface with infinite stiffness. In general, the layer is bounded by two interfaces which both have a finite stiffness, $\tilde{\Sigma}_{1}$ and $\tilde{\Sigma}_{2}$. Then, the interfacial stiffness, $\tilde{\Sigma}_{e f f}$, which enters in the effective Hamiltonian (3.4) for the interfacial separation, $\ell$, is given by ${ }^{8}$

$$
\tilde{\Sigma}_{\text {eff }}=\tilde{\Sigma}_{1} \tilde{\Sigma}_{2} /\left(\tilde{\Sigma}_{1}+\tilde{\Sigma}_{2}\right) \text {. }
$$

Now, consider interaction potentials $V(\ell)$ for the two interfaces which have the form

$$
\begin{array}{rlrl}
V(\ell) & =\infty & \text { for } \quad \ell<0 \\
& =U & \text { for } \quad 0<\ell<\ell_{0} \\
& =W / \ell^{2} \text { for } \ell_{0}<\ell .
\end{array}
$$


It is again convenient to consider rescaled variables

$$
u=2 \tilde{\Sigma}_{\text {eff }} \ell_{0}^{2} U / T^{2} \text { and } w=2 \tilde{\Sigma}_{\text {eff }} W / T^{2}
$$

with $\tilde{\Sigma}_{\text {eff }}$ as in (4.14). In the $(u, w)$ plane, one now finds a line of nontrivial wetting transitions. ${ }^{14}$

This line of transitions consists again of three different subregimes (A), (B), and (C), see Fig. 2. As before, a change in temperature $T$ corresponds to a straight T-trajectory which approaches the origin $(u, w)=(0,0)$ for large $T$. Inspection of Fig. 2 shows that all T-trajectories intersect the transition line at a finite transition temperature, $T=T_{*}<\infty$. This feature is a direct consequence of the fact that the line of wetting transitions does not go through the origin $(u, w)=(0,0)$. This should be compared with the line of roughening transitions in Fig. 1 which contains the origin $(u, w)=(0,0)$.

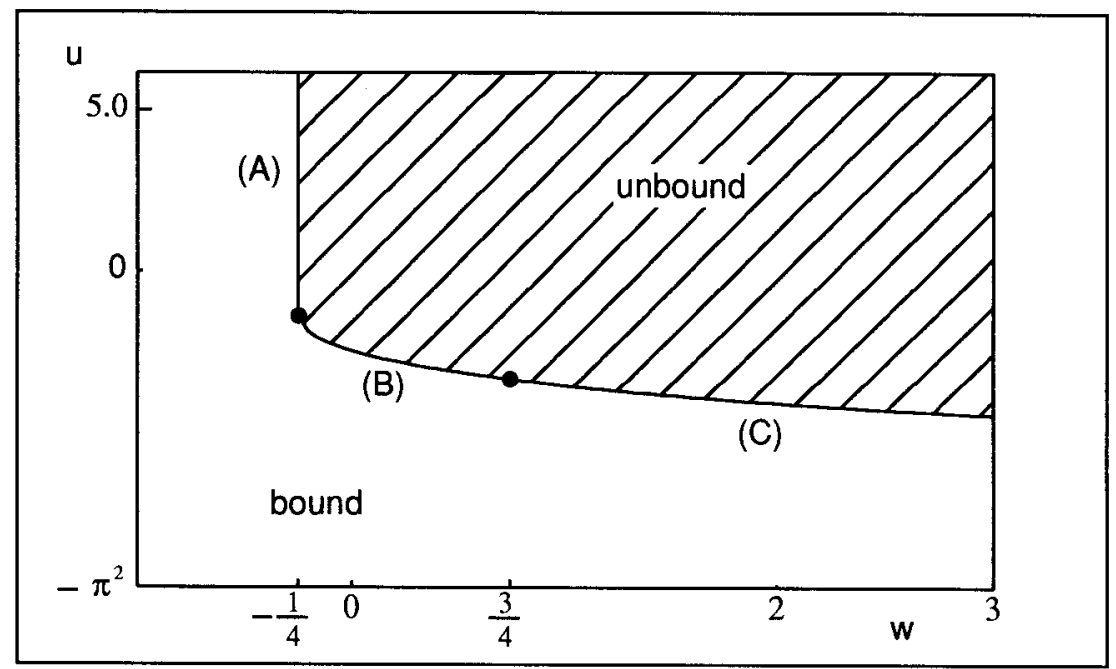

Figure 2: Line of wetting transitions which consists of three regimes (A), (B), and (C). The parameters $w$ and $u$ are defined in (4.16).

The critical behavior in the three subregimes is very similar to the one described in the previous section for roughening. In subregime (A) with $w=-1 / 4$, the mean separation of the two interfaces diverges as

$$
\langle\ell\rangle \sim \exp [\pi / \sqrt{-w-1 / 4}] .
$$

In subregime (B) with $-1 / 4<w<3 / 4$, one has

$$
\langle\ell\rangle \sim 1 /\left(T_{*}-T\right)^{\psi}
$$

with ${ }^{14}$

$$
\psi=1 / \sqrt{1+4 w} .
$$


Subregime (B) contains the case $w=0$ which corresponds to a simple square-well potential as in (4.15) with $W=0$. In this case, one has $\psi=1$ from (4.19) as was first found for the semi-infinite Ising model with a row of weakened surface couplings. In fact, the same critical behavior applies to all attractive potentials which decay faster than $\sim 1 / \ell^{2}$ for large $\ell$.

Finally, in subregime (C) with $w>3 / 4,\langle\ell\rangle$ also diverges as a power law but with the wetting exponent

$$
\begin{array}{rlrl}
\psi & =\frac{1}{2}[3-\sqrt{1+4 w}] & \text { for } 3 / 4<w<2 \\
& =0 & & \text { for } 2<w .
\end{array}
$$

As in the case of roughening transitions, this latter subregime is characterized by exceptional fluctuations and a probability distribution $\widetilde{P}\left(\xi_{\|}^{\zeta}\right) \sim \xi_{\|}^{1-\mu}$ as in (4.13)

\subsubsection{Universal Non-Universality}

Both systems considered in the two preceding sections lead to nonuniversal critical behavior. In both cases, the line of transitions can be parametrized by a dimensionless parameter, $w$.

For roughening from a defect line, this parameter is given by $w=2 W \bar{\Sigma} / T^{2}$ as in (4.3); for wetting, it is defined by $w=2 W \bar{\Sigma}_{1} \bar{\Sigma}_{2} /\left(\tilde{\Sigma}_{1}+\tilde{\Sigma}_{2}\right) T^{2}$ as in (4.16). The critical behavior depends continuously on $w$. In fact, for the above choices of $w$, the critical singularities are identical in both cases even though the transition line differs by a shift parallel to the u-axis, as follows by inspection from Fig. 1 and Fig. 2.

For wetting, an exact functional renormalization group (RG) has been applied which reveals a line of $R G$ fixed points corresponding to the line of wetting transitions. ${ }^{15,16}$ This implies that all potentials which decay as $W / \ell^{2}$ for large $\ell$ exhibit critical singularities which are uniquely determined by $w$ irrespective of the detailed form of the short-ranged part of the potential.

It turns out that this 'universal non-universality' also applies to other systems such as the so-called necklace model. In the latter model, one considers all configuration of $p$ domain boundaries which consist of a 'necklace' of alternating segments, say $A$ and $B$ : In the A segments, all $p$ domain boundaries experience a contact potential and, thus, form a bound state; in the B segments, on the other hand, they experience only a hard wall repulsion and, thus, are essentially free. ${ }^{17,18}$

For $p=3$, the necklace model can be solved for arbitrary stiffness parameters, $\bar{\Sigma}_{1}$, $\bar{\Sigma}_{2}$, and $\tilde{\Sigma}_{3}$ of the three domain boundaries. ${ }^{18}$ The critical singularities found within this model become identical to the singular behavior in the preceding subsection if one makes the identification

$$
w=(\pi / \theta)^{2}-1 / 4
$$

where the angle $\theta$ follows from

$$
\tan \theta=\left[\left(\tilde{\Sigma}_{2} / \tilde{\Sigma}_{1}\right)+\left(\tilde{\Sigma}_{2} / \tilde{\Sigma}_{3}\right)+\left(\tilde{\Sigma}_{2}^{2} / \tilde{\Sigma}_{1} \tilde{\Sigma}_{3}\right)\right]^{1 / 2}
$$

with $0 \leq \theta \leq \pi / 2$.

The necklace model can also be solved for general $p$ and identical stiffness parameters, $\tilde{\Sigma}_{1}=\tilde{\Sigma}_{2}=\ldots=\tilde{\Sigma}_{p} \equiv \tilde{\Sigma}$. In this case, the dimensionless parameter $w$ is given by ${ }^{20}$

$$
w=\left[1-\left(p^{2}-3\right)^{2}\right] / 4 \text {. }
$$

Finally, the same critical behavior is also found for two interacting strings in $d=1+d_{\perp}$ dimensions. If these strings experience short-ranged attractive interactions, one has ${ }^{12}$

$$
w=\frac{1}{4}\left(d_{\perp}-1\right)\left(d_{\perp}-3\right)
$$


for $d_{\perp} \geq 1$.

\subsection{Quasi-periodic systems}

\subsubsection{Roughness and Roughening}

Consider the ideal Penrose tiling which consists of thick and thin rhombi. The edges of these tiles can assume five different directions. For each direction, the tiling can be decomposed into a stack of lanes and rows. The rows consist of all tiles which have two edges parallel to the chosen direction; these rows run, on average, parallel to each other. Two nearest-neighbor rows are separated by one lane. There are two different types of lanes which have a narrow $(\mathrm{N})$ and a wide $(\mathrm{W})$ width, respectively.

It has been realized some time ago that an interface within such a tiling runs, on average, parallel to such a stack of lanes and rows. ${ }^{21,22}$ At zero temperature, it is confined to one of the lanes in which it can assume, however, many degenerate states. This degeneracy is different in the $(\mathrm{N})$ and in the $(\mathrm{W})$ lanes. At finite temperatures, this difference leads to an effective quasi-periodic potential which is approximately given by a Fibonacci potential of the form

$$
\begin{aligned}
V_{Q P}(\ell) & =k_{B} T\left(S_{W}-S_{N}\right) & & \text { for }(\mathrm{N}) \text { lane } \\
& =0 & & \text { for }(\mathrm{W}) \text { lane }
\end{aligned}
$$

where $S_{N} \simeq 0.45$ and $S_{W} \simeq 0.54$ denote the interfacial ground state entropy per step within a $(N)$ and $(W)$ lane of the Penrose tiling, respectively. ${ }^{21}$ If the interface hops across a row, it has to increase its length by one edge and, thus, its energy by twice the nearest-neighbor coupling of the Ising model.

The roughness exponent for this problem has been determined by scaling arguments, real-space renormalization, and numerical studies of the transfer matrix. As a result, one finds that the interface is always rough but that the roughness exponent $\zeta$ is non-universal and depends on the parameters of the model.

The dependence of $\zeta$ on $T / J$ is displayed in Fig. 3 where $J / 2>0$ is the nearest-neighbor coupling in the underlying Ising model. ${ }^{23}$ As shown in Fig. 3, one has $\zeta<1 / 2$ which implies that the quasi-periodic potential leads to a reduction of the roughness. For small $T$, one finds

$$
\zeta \approx 2 \ln (\sigma) T / J \text { with } \sigma=(1+\sqrt{5}) / 2
$$

The Fibonacci-potential is piece-wise constant. Now, consider another type of quasiperiodic potential given by

$$
V_{Q P}(\ell)=-V \cos (2 \pi \ell / \sigma)
$$

which defines the almost-Mathieu or Harper's potential. In this case, the interface undergoes a roughening transition from a smooth state at low $T$ to a rough state at large $T{ }^{21}$ The latter state is characterized by a free interface with $\zeta=1 / 2$. At the roughening transition, on the other hand, the roughness exponent is $\zeta=0.42$ and thus smaller than $1 / 2$. As the roughening temperature, $T=T_{*}^{*}$, is approached from below, one has ${ }^{21}$

$$
\xi_{\perp} \sim 1 /\left|T_{\star}-T\right|^{\nu_{\perp}} \quad \text { with } \quad \nu_{\perp}=1
$$

In the framework of the renormalization group ( $R G$ ), the non-universal values for the roughness exponent $\zeta$ should correspond to a line of $\mathrm{RG}$ fixed points. Then, the roughening transition within the Harper's potential is governed by one of these fixed points corresponding to $\zeta=0.42$. At this roughening transition, there is a relevant perturbation, the flow of which determines the roughening exponent $\nu_{\perp}=1$. In general, one expects that other quasiperiodic potentials will also lead to roughening transitions governed by different fixed points of the Fibonacci-type. 


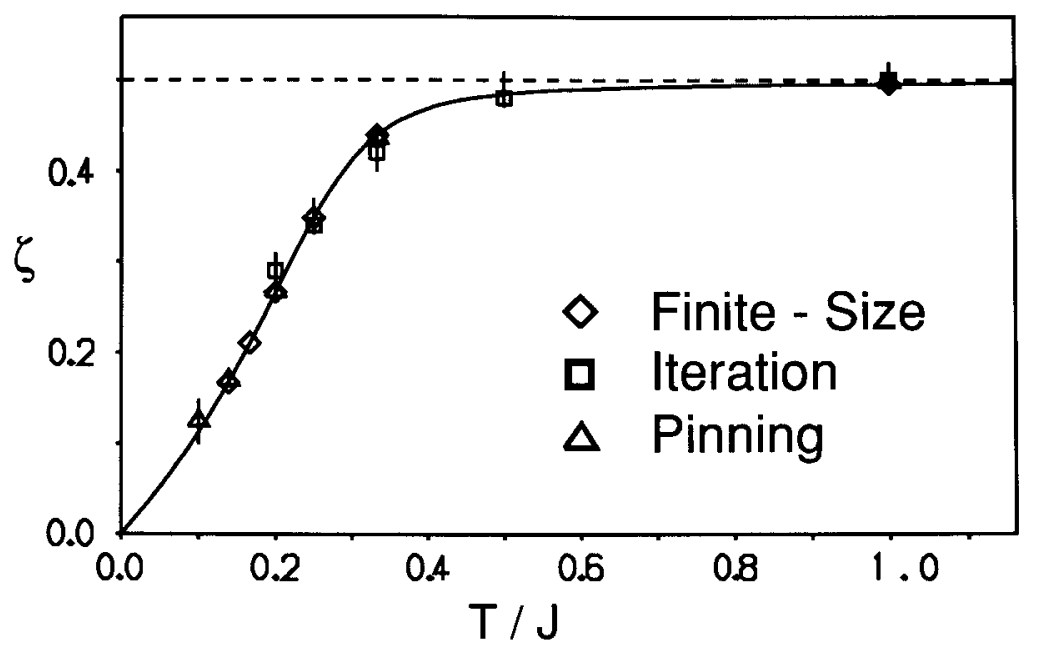

Figure 3: Roughness exponent $\zeta$ for the Fibonacci-potential (4.25) as a function of $\mathrm{T} / \mathrm{J}$.

\subsubsection{Wetting}

Finally, let us briefly discuss the wetting behavior in a quasi-periodic system. ${ }^{23}$ In this case, the interfacial displacement field $\ell$ experiences (i) a quasi-periodic potential, and (ii) an interaction potential as in (4.15). If all forces are short-ranged, this potential has the form

$$
\begin{aligned}
V(\ell) & =\infty & & \text { for } \quad \ell<0 \\
& =U+V_{Q P}(\ell) & & \text { for } 0<\ell<\ell_{0} \\
& =V_{Q P}(\ell) & & \text { for } \ell_{0}<\ell
\end{aligned}
$$

with $U<0$.

In the case where $V_{Q P}(\ell)$ represents a Fibonacci-potential, the critical behavior has been studied by numerical transfer-matrix methods. It is most convenient to probe the wetting transition by changing the depth $U$ of the square-well for fixed Fibonacci-potential. In this case, the system undergoes a wetting transition at a critical depth, $U=U_{*}<0$. The corresponding critical behavior for the parallel correlation length, $\xi_{\|} \sim\langle\ell\rangle^{1 / \zeta} \sim \xi_{1}^{1 / \zeta}$, is shown in Fig. 4. The straight lines represent the result of a three-parameter rms fitting procedure. In this way, one obtains $\nu_{\|} \simeq 1.64,1.25$, and 1.14 for $\zeta=0.439,0.266$, and 0.167 , respectively. ${ }^{23}$ The scaling relation $\psi=\zeta \nu_{\|}$then implies that $\psi \simeq 0.72,0.33$, and 0.19 for these three values of $\zeta$.

For the range of $\zeta$ values which is accessible to the transfer matrix calculations, the critical exponents $\nu_{\|}$and $\psi$ decrease monotonically with decreasing $\zeta$. In fact, these exponents approach the asymptotic values $\nu_{\|}=1$ and $\psi=0$ in the limit of small $\zeta$. This can be understood as follows.

Small $\zeta$ corresponds to low temperatures for which the interface is smooth (or flat). At $T=0$, the wetting behavior follows directly from the potential energy (4.29): for $U<0$, 


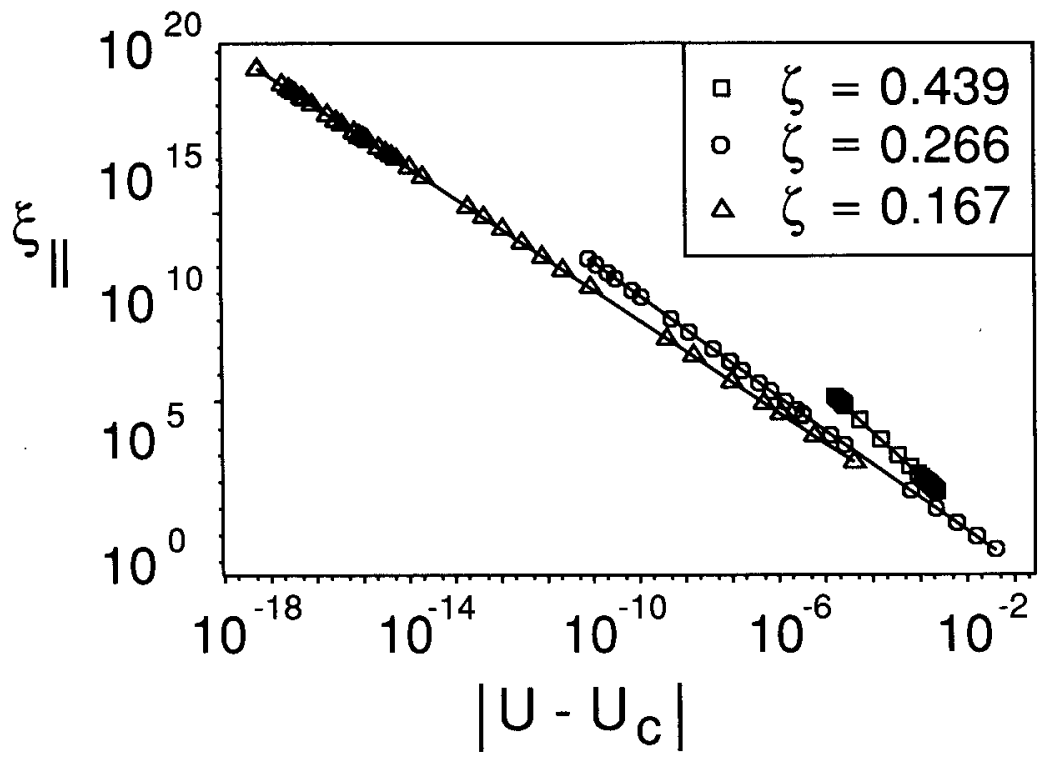

Figure 4: Wetting transitions for the Fibonacci-potential; critical behavior of the parallel correlation length $\xi_{\|}$as a function of $\left|U-U_{*}\right|$ for three different values of $\zeta$ corresponding to three different $\mathrm{T} / \mathrm{J}$.

the interface sits inside the square-well potential; for $U>0$, it sits in one of the minima of the Fibonacci-potential (which are all degenerate). Thus, the interface undergoes a discontinuous transition at $U=0$, and the interfacial (free) energy $f_{s}$ per unit length behaves as $f_{s} \sim|U|$ for small $U$. In general, one has a singular contribution $f_{s} \sim\left|U-U_{*}\right|^{\tau \psi}$ and a regular contribution $\sim\left|U-U_{*}\right|$. Therefore, the transition becomes first-order or discontinuous for $\tau \psi=1 .{ }^{24}$ In the present case, one has $\tau=1 / \zeta$ which implies $\psi / \zeta \approx 1$ or $\psi \approx \zeta$ in the limit of low $T$ or small $\zeta$. In addition, the scaling relation $\psi=\zeta \nu_{\|}$implies $\nu_{\|} \approx 1$ in the same limit.

The critical exponents obtained from the transfer matrix calculation can be reproduced, to within a few percent, by a generalized necklace model. In this model, one obtains the values $^{4}$

$$
\psi=\zeta /(1-\zeta) \text { and } \nu_{\|}=1 /(1-\zeta)
$$

which behave as $\psi \approx \zeta+\zeta^{2}$ and $\nu_{\|} \approx 1+\zeta$ for small $\zeta$. These expressions agree quite well with the numerically obtained values even though the deviations seem to extend the numerical errors. ${ }^{23}$

Finally, it is interesting to point out that the critical behavior of interfaces in Penrose tilings could be studied experimentally in monolayers adsorbed on the facets of decagonal quasi-crystals. For example, one could investigate the behavior of a monolayer of small adatoms. These layers should exhibit 2-dimensional solid, liquid, and vapor phases. As one 
approaches the triple point along the coexistence curve of the solid and the vapor phase, one may observe edge melting, i.e., wetting of the domain boundaries between solid and vapor domains by the liquid phase. If the monolayer sits on top of a decagonal quasi-crystal, the thickening of the wetting layers will reflect the underlying quasi- periodicity.

\section{References}

/1/ For reviews, see R. Lipowsky, in Random fluctuations and patterns growth, ed. by H.E. Stanley and N. Ostrowsky (Kluwer Academic, Dordrecht 1988) p. 227; and G. Forgacs, R. Lipowsky, and T.M. Nieuwenhuizen, in Phase transitions and Critical Phenomena, Vol. 14, ed. by C. Domb and J. Lebowitz (Academic Press, London 1991).

/2/ C. Herring, in Structure and properties of solid surfaces, ed. by R. Gomer and C.S. Smith (Univ. of Chicago press, Chicago 1953).

/3/ A recent review is in H. van Beijeren and I. Nolden, in Structure and Dynamics of Surfaces II, ed. by W. Schommers and P. van Blanckenhagen (Springer-Verlag, 1987).

/4/ R. Lipowsky and C. Henley, Phys. Rev. Lett. 60, 2394 (1988); and R. Lipowsky, in Fundamental problems in statistical mechanics VII, ed. by H. van Beijeren (NorthHolland, Amsterdam 1990) p. 139.

/5/ See, e.g., J.S. Rowlinson and B. Widom, Molecular theory of capillary (Clarendon Press, Oxford 1982).

/6/ See, e.g., R. Pandit, M. Schick and M. Wortis, Phys. Rev. B26, 5112 (1982).

17/ See, e.g., P.G. de Gennes, Rev. Mod. Phys. 57, 827 (1985).

/8/ R. Lipowsky, Phys. Rev. Lett. 52, 1429 (1984); and Phys. Rev. B32, 1731 (1985).

/9/ R. Lipowsky and M.E. Fisher, Phys. Rev. B36, 2126 (1987).

/10/ See, e.g., Surface $x$-ray and neutron scattering, ed. by H. Zabel and I.K. Robinson (Springer Verlag, 1992).

/11/ S. Grotehans and R. Lipowsky, Phys. Rev. A (in press).

/12/ R. Lipowsky, Europhys. Lett. 15, 703 (1991).

/13/ D.B. Abraham, Phys. Rev. Lett. 44, 1165 (1980).

/14/ R. Lipowsky and T.M. Nieuwenhuizen, J. Phys. A21, L89 (1988); R.K.P. Zia, R. Lipowsky, and D.M. Kroll, Am. J. Phys. 56, 160 (1988).

/15/ F. Jülicher, R. Lipowsky, and H. Müller-Krumbhaar, Europhys. Lett. 11, 657 (1990).

/16/ H. Spohn, Europhys. Lett. 14, 689 (1991).

/17/ M.E. Fisher, J. Stat. Phys. 34, 667 (1984).

/18/ D.A. Huse and M.E. Fisher, Phys. Rev. B29, 239 (1984).

/19/ M.E. Fisher and M. Gelfand, J. Stat. Phys. 53, 175 (1988).

/20/ R. Lipowsky, Physica A 177, 182 (1991) 
/21/ C. Henley and R. Lipowsky, Phys. Rev. Lett. 59, 1679 (1987).

/22/ A. Garg and D. Levine, Phys. Rev. Lett. 591683 (1987).

/23/ G.J.O. Schmidt and R. Lipowsky, Europhys. Lett. (submitted).

/24/ R. Lipowsky, Phys. Rev. Lett. 62, 704 (1989). 